\title{
Propagation velocities of chemical reaction fronts advected by Poiseuille flow
}

\author{
Boyd F. Edwards \\ Department of Physics, West Virginia University, Morgantown, West Virginia 26506-6315
}

(Received 25 October 2005; accepted 10 September 2006; published online 12 October 2006)

\begin{abstract}
Poiseuille flow between parallel plates advects chemical reaction fronts, distorting them and altering their propagation velocities. Analytical solutions of the cubic reaction-diffusion-advection equation resolve the chemical concentration for narrow gaps, wide gaps, and small-amplitude flow. Numerical solutions supply a general description for fluid flow in the direction of propagation of the chemical reaction front, and for flow in the opposite direction. Empirical relations for the velocity agree with numerical solutions to within a few percent, and agree exactly with the analytical limits. Applications to nonlinear fingering are discussed. (c) 2006 American Institute of Physics.
\end{abstract}

[DOI: $10.1063 / 1.2358954]$

Chemical waves generally alter the mass densities of the aqueous solutions through which they propagate. These density changes can lead to buoyancy-driven convection. When bounded by parallel no-slip plates, such convection has a local velocity profile which peaks at the gap center and is zero at the walls. Such nonuniform "Poiseuille" flow distorts chemical reaction fronts and alters their velocities of propagation. The purpose of the study is to investigate these effects using the Navier-Stokes equations and the cubic reaction-diffusion-advection equation pertinent to the iodate-arsenous acid reaction. In contrast with previous assumptions, the propagation velocity is found to exceed the sum of the velocity of a planar front in a static fluid and the average flow velocity. Velocity results preclude the need for the reaction-diffusionadvection equation in future studies of nonlinear fingering.

\section{INTRODUCTION}

Nonuniform fluid flow alters the shapes and propagation velocities of chemical reaction fronts through the reactiondiffusion-advection equation ${ }^{1-6}$

$$
\frac{\partial C}{\partial t}+\mathbf{V} \cdot \nabla C=D_{C} \nabla^{2} C-F(C)
$$

From right to left, the terms in Eq. (1) give the chemical reaction rate $F(C)$, the molecular diffusion rate, the rate of advection by the fluid flow, and the resulting rate of change in the local chemical concentration $C(\mathbf{x}, t)$. The advection term alters the propagation velocity of chemical reaction fronts and distorts them when the fluid velocity $\mathbf{V}(\mathbf{x}, t)$ is nonuniform.

The Navier-Stokes equations demand that twodimensional (2D) flow between parallel no-slip plates at $x$ $= \pm a / 2$ (a "Hele-Shaw" cell) assume the quadratic Poiseuille velocity profile ${ }^{7}$

$$
\mathbf{V}(x, y, z, t)=\frac{3}{2}\left(1-\frac{4 x^{2}}{a^{2}}\right) \overline{\mathbf{V}}(y, z, t),
$$

with gap average velocity $\overline{\mathbf{V}}$, with peak velocity $(3 / 2) \overline{\mathbf{V}}$ (reached at the gap center $x=0$ ), and with $\hat{\mathrm{x}} \cdot \overline{\mathbf{V}}=0$. To simplify calculations for small gaps and small-amplitude flows, $\mathbf{V}$ is often replaced by $\overline{\mathbf{V}}$ and the $x$ dependence of $C$ is ignored in Eq. (1). ${ }^{2,3,8}$ These approximations, which are not made herein, imply a local front velocity $U=U_{0}+W$ that equals the sum of the velocity $U_{0}$ of a planar front propagating through a static fluid and the component $W=\hat{p} \cdot \overline{\mathbf{V}}$ of the average fluid velocity in the direction $\hat{p}$ of propagation of the front in the $y-z$ plane.

For simplicity, we consider the iodate-arsenous acid reaction with the cubic reaction rate $e^{1-3}$

$$
F(C)=\alpha C\left(C-C_{2}\right)\left(C-C_{3}\right),
$$

which conveniently admits a closed-form solution to Eq. (1) with propagation velocity $U_{0}=\left(\alpha D_{C} / 2\right)^{1 / 2} C_{2} \approx 0.03 \mathrm{~mm} / \mathrm{s}$. ${ }^{1}$ The iodate-arsenous acid reaction produces its own catalyst, whose molecular diffusion into the unreacted fluid limits this velocity. The autocatalyst concentration $C$ increases monotonically from its initial value of zero far ahead of the propagating front to its final value $C_{2}$ far behind, and increases steeply in a narrow region of thickness $L_{0}=D_{C} / U_{0}$ $\approx 0.07 \mathrm{~mm}$, called the reaction front, where much of the chemical reaction takes place. The constant $\alpha$ governs the overall reaction rate and $C_{3}$ is a small negative ratio of rate constants.

Iodate-arsenous acid fronts convert an unreacted fluid mixture into a less-dense reacted fluid mixture, and are therefore potentially unstable to buoyancy-driven convection when the reaction proceeds upward. Indeed, flat fronts ascending in vertical slabs bounded by parallel vertical no-slip plates are unstable to buoyancy-driven convection above a critical gap width. ${ }^{2,3,9-15}$ For these studies, the Navier-Stokes equations are used in conjunction with the reactiondiffusion-advection equation to determine the flow resulting 
from the mass density gradients associated with the chemical reactions. Since small-amplitude flows near the onset of convection do not significantly distort the reaction front, calculations of the onset of convection which replace $\mathbf{V}$ by $\overline{\mathbf{V}}$ and that ignore the $x$ dependence of $C$ are justified. ${ }^{2,3}$ However, finite-amplitude flows above the onset of convection distort ascending reaction fronts and alter their velocities of propagation. These effects may be important in experiments on nonlinear fingering. ${ }^{11}$ Although experiments on ascending reaction fronts may require buoyancy-driven three-dimensional (3D) modifications of the Poiseuille profile for wide gaps, descending reaction fronts in wide gaps require no such modifications. ${ }^{12}$ Thus, experiments on the response of descending iodate-arsenous acid fronts to imposed Poiseuille flow in a Hele-Shaw cell would be valuable because they would isolate the effects of the Poiseuille flow from buoyancy-driven modifications.

When $L_{0} \ll a$, Eqs. (1) and (3) reduce to the eikonal equation $^{16-18}$

$$
U_{n}=U_{0}+D_{C} K+V_{n}
$$

governing the normal component of velocity $U_{n}$ of a curved advected surface of constant concentration, where $V_{n}=\hat{n} \cdot \mathbf{V}$ is the normal component of fluid velocity, $\hat{n}$ is the unit normal vector pointing into the unreacted fluid, and $K=-\boldsymbol{\nabla} \cdot \hat{n}$ is the curvature, which is positive when the center of curvature is in the unreacted fluid. In Ref. 18, we show that Eq. (4), normally considered to be valid only when the front thickness is small compared to the gap width, is also valid in the opposite limit. A midgap cusp in the reaction front that we predicted earlier for wide-gap adverse flows ${ }^{8}$ was subsequently observed experimentally. ${ }^{19}$

In this paper, we present numerical and analytical calculations of concentration profiles and front shapes and construct empirical expressions for the front velocity that match our numerical calculations. These empirical expressions preclude the need to include the full reaction-diffusionadvection equation in future investigations of nonlinear fingering. In particular, we present detailed analytical solutions for $U$ in a $2 \mathrm{D}$ slot defined by $-a / 2 \leq \mathbf{x} \leq a / 2$ and $-\infty<z$ $<\infty$, with no $y$ dependence $(\partial C / \partial y=0)$ and steady Poiseuille flow in the $\hat{p}=\hat{z}$ direction with $\overline{\mathbf{V}}=W \hat{z}$ and $W=$ const (see Table I). Equation (2) accordingly reduces to

$$
\mathbf{V}(x)=\frac{3}{2}\left(1-\frac{4 x^{2}}{a^{2}}\right) W \hat{z} .
$$

We consider the small-amplitude limit (Sec. III), the narrowgap limit (Sec. IV), and the wide-gap limit (Sec. V), and solve the general problem numerically (Sec. VI). We also construct an empirical closed-form solution (Sec. VII) for $U$ that agrees exactly with all analytical limits, and which reproduces all numerical results to within a few percent.

We restrict spatial gradients in the front height to the direction perpendicular to the confining plates at $x= \pm a / 2$, and accordingly denote the height of the front surface by $z$ $=H(x, t)$. Here we do not consider spatial variations in the

\begin{tabular}{|c|c|}
\hline $\bar{a}$ & Gap width \\
\hline$C(x, z, t)$ & Catalyst concentration \\
\hline$C_{2}$ & $\begin{array}{l}\text { Final catalyst concentration (long after passage } \\
\text { of the chemical front) }\end{array}$ \\
\hline$C_{3}$ & Small negative ratio of rate constants \\
\hline$c(x, z, t)=C(x, z, t) / C_{2}$ & Dimensionless catalyst concentration \\
\hline$D_{C}$ & Catalyst molecular diffusivity \\
\hline$H(x, t)$ & Front height, $z=H(x, t)$ \\
\hline$h(x, t)$ & Dimensionless front height, $z=h(x, t)$ \\
\hline$K(x, t)=-\boldsymbol{\nabla} \cdot \hat{n}$ & Front curvature \\
\hline$L_{0}=D_{C} / U_{0}$ & Reaction front thickness \\
\hline$\hat{n}(x, t)$ & $\begin{array}{l}\text { Unit vector normal to the front pointing into the } \\
\text { unreacted fluid }\end{array}$ \\
\hline$\hat{p}=\hat{z}$ & Unit vector in the direction of front propagation \\
\hline $\mathrm{Pe}=W a / 2 D_{C}=\epsilon \eta$ & Peclet number \\
\hline$t$ & Time \\
\hline$u=U / U_{0}$ & Dimensionless front velocity \\
\hline$U$ & Front velocity \\
\hline$U_{0}$ & Front velocity for a planar front in a static fluid \\
\hline$U_{n}(x, t)=\hat{n} \cdot \hat{z} \partial H / \partial t$ & $\begin{array}{l}\text { Component of the local front velocity normal } \\
\text { to the front }\end{array}$ \\
\hline$W=\hat{p} \cdot \overline{\mathbf{V}}$ & $\begin{array}{l}\text { Gap-averaged Poiseuille velocity in the direction } \\
\text { of propagation }\end{array}$ \\
\hline $\mathbf{V}(x)$ & Poiseuille velocity profile \\
\hline$\overline{\mathbf{V}}$ & Gap-averaged Poiseuille velocity \\
\hline$V_{n}=\hat{n} \cdot \mathbf{V}$ & Normal component of Poiseuille velocity \\
\hline$x$ & Cross gap Cartesian coordinate \\
\hline$z$ & $\begin{array}{l}\text { Cartesian coordinate in the direction of front } \\
\text { propagation }\end{array}$ \\
\hline$\alpha$ & Reaction rate constant \\
\hline$\epsilon=W / U_{0}$ & $\begin{array}{l}\text { Dimensionless average Poiseuille velocity in the } \\
\text { propagation direction }\end{array}$ \\
\hline$\eta=a / 2 L_{0}$ & Ratio of gap half width to front thickness \\
\hline$\zeta=\eta(z-u t)$ & Comoving dimensionless coordinate \\
\hline
\end{tabular}

TABLE I. Fundamental variables used in this paper.

front height in the horizontal $(y)$ direction parallel to the plates, but expect our results to be applicable in this case. Accordingly,

$$
\hat{n}=\frac{\hat{z}-\hat{x} \partial H / \partial x}{\left[1+(\partial H / \partial x)^{2}\right]^{1 / 2}}
$$

and $U_{n}=\hat{n} \cdot \hat{z} \partial H / \partial t$ allow us to recast Eq. (4) as a partial differential equation for $H(x, t)$ :

$$
\begin{aligned}
\frac{\partial H}{\partial t}= & U_{0}\left[1+\left(\frac{\partial H}{\partial x}\right)^{2}\right]^{1 / 2}+\frac{3}{2} W\left(1-\frac{4 x^{2}}{a^{2}}\right) \\
& +D_{C}\left[1+\left(\frac{\partial H}{\partial x}\right)^{2}\right]^{-1} \frac{\partial^{2} H}{\partial x^{2}} .
\end{aligned}
$$

Other related studies include the nonlinear interactions of chemical reactions and viscous fingering in porous media, ${ }^{20}$ the effective diffusivity for Poiseuille flow in the absence of chemical reactions, ${ }^{21}$ oscillatory hydrodynamic flow for reaction fronts driven by surface tension gradients, ${ }^{22,23}$ Oregonator model simulations including surface tension and hydrodynamics, ${ }^{24}$ the Rayleigh-Taylor instability of miscible fluids in a Hele-Shaw cell, ${ }^{25}$ and the recent observation of buoyant plumes. ${ }^{26}$ Acidic chloritetetrathionate reaction fronts have a quartic reaction rate and 
convert an unreacted fluid mixture into a more-dense reacted mixture, and are therefore potentially unstable to buoyancydriven convection when the reaction proceeds downward. ${ }^{4,5}$ Our future investigations may include detailed analysis of this reaction.

\section{DIMENSIONLESS EQUATIONS}

It is convenient to define dimensionless variables according to $x=(a / 2) x^{\prime}, \quad z=(a / 2) z^{\prime}, \quad t=\left(a / 2 U_{0}\right) t^{\prime}, \quad$ and $C(x, z, t)=C_{2} c\left(x^{\prime}, z^{\prime}, t^{\prime}\right)$. Substituting Eq. (2) into Eq. (1) and dropping the primes yields

$$
\frac{\partial c}{\partial t}+\frac{3}{2} \epsilon\left(1-x^{2}\right) \frac{\partial c}{\partial z}=\frac{1}{\eta}\left(\frac{\partial^{2} c}{\partial x^{2}}+\frac{\partial^{2} c}{\partial z^{2}}\right)+2 \eta c^{2}(1-c),
$$

where $\epsilon=W / U_{0}$ is the dimensionless component of the average Poiseuille velocity in the direction of propagation of the front, and $\eta=a / 2 L_{0}$ is the ratio of the gap half width to the front thickness. Accordingly, the product of $\epsilon$ and $\eta$ gives the Peclet number $\mathrm{Pe}=\epsilon \eta=W a / 2 D_{C}$, a measure of the importance of advection relative to diffusion. ${ }^{20}$ Since $C_{3} / C_{2} \approx-2$ $\times 10^{-3},{ }^{1,27} C_{3}$ plays an insignificant role in Eq. (1), and we ignore it. Equation (8) governs the evolution of the dimensionless autocatalyst concentration $c(x, z, t)$ in the $x-z$ plane perpendicular to the plates. For propagation in the $+z$ direction, we require $c \rightarrow 0$ as $z \rightarrow+\infty$ and $c \rightarrow 1$ as $z \rightarrow-\infty$. We also require impermeable plates by setting $\partial c / \partial x=0$ at $x$ $= \pm 1$. Constant-concentration front profiles $h_{c}(x)$ aptly describe the shapes of moving surfaces of constant concentration $c$, which satisfy $z=h_{c}(x)+u t$.

Soliton solutions of Eq. (8) propagating without changing shape at a constant, uniform dimensionless velocity $u$ (measured in units of $U_{0}$ ) in the $+z$ direction must have $z$ and $t$ dependences that occur only in the combination $z-u t$. Employing a comoving coordinate $\zeta=\eta(z-u t)$, the associated concentration $c(x, \zeta)$ satisfies

$$
\frac{1}{\eta^{2}} \frac{\partial^{2} c}{\partial x^{2}}+\frac{\partial^{2} c}{\partial \zeta^{2}}+\left[u-\frac{3}{2} \epsilon\left(1-x^{2}\right)\right] \frac{\partial c}{\partial \zeta}+2 c^{2}(1-c)=0,
$$

with the boundary conditions

$$
\begin{aligned}
& c(x,+\infty)=0, \\
& c(x,-\infty)=1, \\
& \frac{\partial c}{\partial x}( \pm 1, \zeta)=0 .
\end{aligned}
$$

Defining dimensionless variables according to $x$ $=(a / 2) x^{\prime}, t=\left(a / 2 U_{0}\right) t^{\prime}$, and $H(x, t)=(a / 2) h\left(x^{\prime}, t^{\prime}\right)$ allows us to write the dimensionless form of the eikonal equation, Eq. (7), as

$$
\begin{aligned}
\frac{\partial h}{\partial t}= & {\left[1+\left(\frac{\partial h}{\partial x}\right)^{2}\right]^{1 / 2}+\frac{3}{2} \epsilon\left(1-x^{2}\right) } \\
& +\eta^{-1}\left[1+\left(\frac{\partial h}{\partial x}\right)^{2}\right]^{-1} \frac{\partial^{2} h}{\partial x^{2}}
\end{aligned}
$$

where we have once again dropped the primes on the dimensionless independent variables, and where $z=h(x, t)$ defines the dimensionless time-dependent position of the front.

For soliton solutions that propagate at a constant, uniform dimensionless velocity $u=\partial h / \partial t$ (measured in units of $U_{0}$ ) in the $+z$ direction, Eq. (13) reduces to the ordinary differential equation

$u=\left[1+\left(h^{\prime}\right)^{2}\right]^{1 / 2}+\frac{3}{2} \epsilon\left(1-x^{2}\right)+\eta^{-1}\left[1+\left(h^{\prime}\right)^{2}\right]^{-1} h^{\prime \prime}$,

where the time-dependent front position $z=h(x)+u t$ involves the dimensionless front profile $h(x)$. Impermeable boundaries demand that $h^{\prime}( \pm 1)=0$. Equations (13) and (14) apply in the limit of large $\eta$.

In the sections that follow, Eqs. (8)-(14) serve as the basis for a variety of approaches to the problem of Poiseuille advection of chemical reaction fronts.

\section{SMALL-AMPLITUDE FLOW LIMIT; $\epsilon \rightarrow 0$}

When $\epsilon \ll 1$, the Poiseuille flow velocity is small compared with the velocity of propagation of the reaction front. To seek soliton solutions of Eqs. (9)-(12) for such smallamplitude Poiseuille flow, we expand in powers of $\epsilon$ according to

$$
\begin{aligned}
& c=c_{0}+c_{1}+\cdots, \\
& u=u_{0}+u_{1}+\cdots,
\end{aligned}
$$

with $\eta$ of order unity. To zeroth order, Eqs. (9)-(12) require

$$
\frac{1}{\eta^{2}} \frac{\partial^{2} c_{0}}{\partial x^{2}}+\frac{\partial^{2} c_{0}}{\partial \zeta^{2}}+u_{0} \frac{\partial c_{0}}{\partial \zeta}+2 c_{0}^{2}\left(1-c_{0}\right)=0,
$$

$c_{0}(x,+\infty)=0, c_{0}(x,-\infty)=1$, and $\partial c_{0}( \pm 1, \zeta) / \partial x=0$. These are satisfied by $u_{0}=1$ and

$$
c_{0}(\zeta)=\frac{1}{1+e^{\zeta}}
$$

the celebrated soliton solution for a flat front propagating with speed $U_{0}$ (in conventional units) through a static fluid. ${ }^{1}$ For this solution, the value $\zeta=0$ identifies the surface of constant concentration $c=1 / 2$. This concentration is midway between the initial and final concentrations $c=0$ (at $\zeta \rightarrow \infty$ ) and $c=1($ at $\zeta \rightarrow-\infty)$, and accordingly defines the middle of the reaction front, where the chemical reaction is $50 \%$ complete.

To first order, Eqs. (9)-(12) require

$$
\begin{gathered}
\frac{1}{\eta^{2}} \frac{\partial^{2} c_{1}}{\partial x^{2}}+\frac{\partial^{2} c_{1}}{\partial \zeta^{2}}+u_{0} \frac{\partial c_{1}}{\partial \zeta}+2 c_{0}\left(2-3 c_{0}\right) c_{1} \\
=\left[\frac{3}{2} \epsilon\left(1-x^{2}\right)-u_{1}\right] \frac{\partial c_{0}}{\partial \zeta},
\end{gathered}
$$

$c_{1}(x, \pm \infty)=0$, and $\partial c_{1}( \pm 1, \zeta) / \partial x=0$. The inhomogeneity on the right-hand side of Eq. (19) suggests a solution of the form

$$
c_{1}(x, \zeta)=-\eta h(x) \frac{\partial c_{0}}{\partial \zeta},
$$

which satisfies the boundary conditions as long as $h^{\prime}( \pm 1)$ $=0$. Substituting this form into Eq. (19), integrating once with respect to $\zeta$, and applying Eq. (17) leaves 


$$
h^{\prime \prime}=\eta u_{1}-\frac{3}{2} \epsilon \eta\left(1-x^{2}\right) .
$$

Integrating with respect to $x$ and applying $h^{\prime}( \pm 1)=0$ gives $u_{1}=\epsilon$ and

$$
h(x)=\frac{\epsilon \eta}{8}\left(1-x^{2}\right)^{2},
$$

where we have chosen $h( \pm 1)=0$.

Combining the results above, we obtain the concentration

$$
c(x, z, t)=\frac{1}{1+e^{\eta[z-u t-h(x)]}},
$$

and speed

$$
u=1+\epsilon
$$

of an advected reaction front that propagates without change of shape, valid through first order in the advection strength $\epsilon$. Since $z=h(x)+u t$ gives the position of a surface of constant concentration $c=1 / 2, h(x)$ is just the front profile associated with the soliton eikonal equation, Eq. (14).

\section{NARROW-GAP LIMIT; $\boldsymbol{\eta} \rightarrow 0$}

It is also useful to explore soliton solutions of Eqs. (9)-(12) for $\eta \ll 1$, that is, when the gap width is small compared with the thickness of the reaction front. Expanding now in even powers of $\eta$, we write

$$
\begin{aligned}
& c=c^{(0)}+c^{(2)}+\cdots, \\
& u=u^{(0)}+u^{(2)}+\cdots,
\end{aligned}
$$

with $\epsilon$ of order unity.

To order $\eta^{-2}$, Eq. (9) gives

$$
\frac{1}{\eta^{2}} \frac{\partial^{2} c^{(0)}}{\partial x^{2}}=0 \text {. }
$$

Integrating once with respect to $x$ and applying $\partial c^{(0)}( \pm 1, \zeta) / \partial x=0$ [Eq. (12)] reveals that $c^{(0)}$ is independent of $x$.

To order $\eta^{0}$, Eq. (9) gives

$$
\begin{aligned}
-\frac{1}{\eta^{2}} \frac{\partial^{2} c^{(2)}}{\partial x^{2}}= & \frac{\partial^{2} c^{(0)}}{\partial \zeta^{2}}+\left[u^{(0)}-\frac{3}{2} \epsilon\left(1-x^{2}\right)\right] \frac{\partial c^{(0)}}{\partial \zeta} \\
& +2 c^{(0) 2}\left(1-c^{(0)}\right) .
\end{aligned}
$$

Integrating once with respect to $x$ gives

$$
\begin{aligned}
-\frac{1}{\eta^{2}} \frac{\partial c^{(2)}}{\partial x}= & {\left[\frac{\partial^{2} c^{(0)}}{\partial \zeta^{2}}+\left(u^{(0)}-\frac{3}{2} \epsilon+\frac{1}{2} \epsilon x^{2}\right) \frac{\partial c^{(0)}}{\partial \zeta}\right.} \\
& \left.+2 c^{(0) 2}\left(1-c^{(0)}\right)\right] x+A(\zeta)
\end{aligned}
$$

Applying $\partial c^{(2)}( \pm 1, \zeta) / \partial x=0[$ Eq. $(12)]$ gives $A(\zeta)=0$ and requires

$$
\frac{\partial^{2} c^{(0)}}{\partial \zeta^{2}}+\left(u^{(0)}-\epsilon\right) \frac{\partial c^{(0)}}{\partial \zeta}+2 c^{(0) 2}\left(1-c^{(0)}\right)=0 .
$$

This equation has a soliton solution

$$
\begin{aligned}
& c^{(0)}=\frac{1}{1+e^{\zeta}}, \\
& u^{(0)}=1+\epsilon,
\end{aligned}
$$

which satisfies the remaining boundary conditions, and which is valid for small $\eta$ and finite $\epsilon$. This result is identical to Eq. (24), which is valid instead for finite $\eta$ and small $\epsilon$. This agreement is consistent with the fact that the $\eta \rightarrow 0$ and the $\epsilon \rightarrow 0$ limits both correspond to weak flows with small Peclet numbers $\mathrm{Pe}=\epsilon \eta$.

Integrating Eq. (29) with respect to $x$ gives

$$
c^{(2)}(x, \zeta)=-\eta[h(x)+B(\zeta)] \frac{\partial c^{(0)}}{\partial \zeta},
$$

where $h(x)$ is the front profile given by Eq. (22), and where the second term is an integration "constant" with respect to $x$, and involves a nonzero undetermined function $B(\zeta)$. To determine $B(\zeta)$ requires the order- $\eta^{2}$ solution of Eq. (9), which has thus far eluded our analytical skill.

Combining the results above, we obtain the concentration

$$
c(x, z, t)=\frac{1}{1+e^{\eta[z-u t-h(x)-B(\zeta)]}},
$$

valid through second order in $\eta$. Apart from an additive function of $\zeta=\eta(z-u t)$ in the exponential, this result agrees with Eq. (23). Accordingly, the concentration profile $h(x)$ at fixed $\zeta$ is valid both through second order in $\eta$ and through first order in $\epsilon$.

\section{WIDE-GAP LIMIT; $\boldsymbol{\eta} \rightarrow \infty$}

Equations (9)-(12) are intractable for $\eta \rightarrow \infty$, that is, for gaps that are wide compared with the front thickness. Fortunately, useful analytical results can be obtained in this limit from Eq. (14), the eikonal equation. The $\eta \rightarrow \infty$ limit of Eq. (14) is singular because the coefficient of the highest-order derivative vanishes in this limit. Ignoring the second-order curvature term gives a first-order equation

$$
u=\left[1+\left(h^{\prime}\right)^{2}\right]^{1 / 2}+\frac{3}{2} \epsilon\left(1-x^{2}\right),
$$

which is valid except where $h^{\prime \prime}$ is of order $\eta$. The profile $h(x)$ for a front that propagates steadily without change of shape must be even about $x=0$ because the Poiseuille flow is even. We can therefore focus on the interval $0 \leq x \leq 1$, with the boundary conditions $h^{\prime}(0)=h^{\prime}(1)=0$. Only one of these conditions can be satisfied by choosing the eigenvalue $u$ in Eq. (35), with the sign of $\epsilon$ determining which one. To satisfy the other condition at the "singular" boundary requires the curvature term in Eq. (14), as will be seen.

Equation (35), a first-order ordinary differential equation, cannot generally satisfy the two boundary conditions $h^{\prime}(0)=h^{\prime}(1)=0$. This is the standard conundrum encountered in singular boundary problems. ${ }^{28}$ In such problems, the coefficient of the highest-order derivative vanishes in the limit of interest, thereby reducing the order of the differential equation by at least one, and reducing the number of boundary conditions that can be satisfied generally. In our case, the 
coefficient of the $h^{\prime \prime}(x)$ term in Eq. (14) vanishes in the limit $\eta \rightarrow \infty$, reducing this equation to a first-order equation, Eq. (35). In this limit, the solution of Eq. (35) is called the lowest-order "outer" solution in singular perturbation theory ${ }^{28}$ and is valid everywhere except at one of the boundaries, called the singular boundary, where $h^{\prime \prime}(x)$ is of order $\eta$. For $\epsilon>0$, the singular boundary is $x= \pm 1$ and for $\epsilon<0$, the singular boundary is $x=0$.

Singular perturbation theory can be used to find analytical solutions near singular boundaries, called "inner" solutions, which can be matched with the outer solutions to provide complete solutions in the singular limit. ${ }^{28}$ Our numerical solutions for large but finite $\eta$ anticipate the behavior at the singular boundaries as $\eta \rightarrow \infty$. In Fig. 3 of Ref. 8 , the curvature $h^{\prime \prime}(x)$ near $x= \pm 1$ clearly increases with increasing $\eta$, anticipating the infinite curvature associated with the singular limit. In Fig. 4 of Ref. 8, the curvature near $x$ $=0$ behaves similarly. Thus, our two independent approaches to the singular problem, numerical solution of Eq. (9) and analytical solution of Eq. (14), yield solutions that complement and agree with each other.

\section{A. Poiseuille flow in the direction of the chemical reaction; $\epsilon>0$}

For $\epsilon>0$, the uniformity of $u$ in Eq. (35) demands that $\left|h^{\prime}\right|$ increase as $x$ increases from 0 to 1 . Accordingly, Eq. (35) can satisfy only the boundary condition $h^{\prime}(0)=0$, which requires

$$
u=1+\frac{3}{2} \epsilon .
$$

Inserting this result into Eq. (35) and integrating gives the outer solution

$$
h(x)=D-\frac{\epsilon}{2}\left(\frac{4}{3 \epsilon}+x^{2}\right)^{3 / 2},
$$

valid everywhere except inside a thin boundary layer at the singular boundary at $x=1$. The thickness of this layer is of order $1 / \eta$, as will be shown below.

The inner solution $h_{s}(x)$ that is valid near this singular boundary obeys

$$
\frac{d^{2} h_{s}}{d x^{2}}=\frac{3}{2} \epsilon \eta,
$$

obtained by setting $h=h_{s}$ and $h_{s}^{\prime}=0$ in Eq. (14) and by inserting Eq. (36). Integrating once and requiring $h_{s}^{\prime}(1)=0$ yields

$$
\frac{d h_{s}}{d x}=\frac{3}{2} \epsilon \eta(x-1) .
$$

Integrating again and choosing $h_{s}(1)=0$ gives

$$
h_{s}(x)=\frac{3 \epsilon \eta}{4}(1-x)^{2} .
$$

We now match the solutions given by Eqs. (37) and (49) by seeking the values of $D$ and $x=1-\delta$ for which $h(x)$ $=h_{s}(x)$ and $h^{\prime}(x)=h_{s}^{\prime}(x)$. Expanding in powers of $\eta^{-1}$ according to

$$
D=D_{0}+\eta^{-1} D_{1}+\cdots,
$$

$$
\delta=\delta_{0}+\eta^{-1} \delta_{1}+\cdots
$$

gives

$$
\begin{aligned}
& D=\frac{\epsilon \beta^{3}}{2}\left(1-\frac{3}{2 \beta \eta}\right), \\
& \delta=\frac{\beta}{\eta}
\end{aligned}
$$

through first order, where we define

$$
\beta=\left(1+\frac{4}{3 \epsilon}\right)^{1 / 2}
$$

for convenience. Equation (44) ensures that the boundarylayer thickness $\delta$ becomes small in the wide-gap limit $\eta$ $\rightarrow \infty$.

In summary, for $\eta \rightarrow \infty$ and $\epsilon>0$, advected chemical reaction fronts propagate in the $+z$ direction with velocity

$$
u=1+\frac{3}{2} \epsilon,
$$

with the "outer" profile

$$
h(x)=\frac{\epsilon}{2}\left[\beta^{3}-\left(\frac{4}{3 \epsilon}+x^{2}\right)^{3 / 2}\right]-\frac{3 \epsilon \beta^{2}}{4 \eta}
$$

for $|x|<\beta / \eta$, and with the "inner" profile

$$
h(x)=\frac{3 \epsilon \eta}{4}(1-|x|)^{2}
$$

for the singular boundary layer $\beta / \eta<|x|<1$, where $\beta$ is given by Eq. (45). Accordingly, front curvature is appreciable only at the thin boundary layer, where it ensures the satisfaction of the boundary condition $h^{\prime}( \pm 1)=0$.

\section{B. Poiseuille flow in the direction opposite the chemical reaction; $\epsilon<0$}

For $\epsilon<0$, the uniformity of $u$ in Eq. (35) demands that $\left|h^{\prime}\right|$ decrease with increasing $x$. Accordingly, Eq. (35) can satisfy only the boundary condition $h^{\prime}(1)=0$, which requires

$$
u=1 .
$$

Inserting this result into Eq. (35) leads to an integral

$$
h(x)=-\frac{3|\epsilon|}{2} \int_{|x|}^{1}\left(1-x^{2}\right)^{1 / 2}\left(1+\frac{4}{3|\epsilon|}-x^{2}\right)^{1 / 2} d x,
$$

which is challenging for general $\epsilon$, but which is easily evaluated numerically. The integral is nevertheless elementary for $|\epsilon| \rightarrow 0$, giving

$$
h(x)=\frac{\sqrt{3|\epsilon|}}{2}\left[|x|\left(1-x^{2}\right)^{1 / 2}+\sin ^{-1}|x|-\pi / 2\right],
$$

and for $|\epsilon| \rightarrow \infty$, giving

$$
h(x)=\frac{|\epsilon|}{2}\left(3|x|-|x|^{3}-2\right),
$$

where $-1<x<1$ and $h(1)=0$ in both cases. These solutions are valid except inside a thin boundary layer at the cusp 
singularity at $x=0$. The thickness of this layer is of order $1 / \eta$. The singular solution very near this cusp may be found and matched using the procedure outlined above for $\epsilon>0$, if needed.

\section{NUMERICAL SIMULATIONS}

To explore results for intermediate $\eta$ and finite $\epsilon$, we perform finite-difference simulations of Eq. (8) on a rectangular computational domain defined by $-1 \leq x \leq 1$ and 0 $\leq z \leq b$, which is divided into a grid with lattice spacing $\Delta x$ in the $x$ and $z$ directions. To accurately represent a domain that is unbounded in the $\pm z$ directions by a computational domain that is finite, and to avoid the need to recenter the front on the computational domain when the front approaches the upper computational boundary, periodic boundary conditions are employed to equate values of $c$ at the $z$ $=0$ and the $z=b$ boundaries, and a moving physical boundary is defined at $z_{b}=z_{0}+b / 2$, where $z_{0}$ is the current location of the center of the front. We set $c=0$ at $z=z_{b}$ to simulate the boundary at $z=+\infty$, and set $c=1$ at $z=z_{b}+\Delta x$ to simulate the boundary at $z=-\infty$. The front repeatedly traverses the computational domain, with $z_{0}$ following the front as it moves and with $z_{b}=z_{0}+b / 2$ located half the domain width ahead of the front. Setting $b=2 r+h_{1 / 2}(1)-h_{1 / 2}(0)$ ensures a decay distance $r$ ahead of the front for the concentration to decay to zero and a distance $r$ behind the front for the concentration to approach unity. Equation (23) indicates that the approach to these values is exponential, with an exponential decay distance of $\eta^{-1}$. Accordingly, we find that $r=10 / \eta$ provides an adequate description, and that using $r=20 / \eta$ yields corrections that alter the front velocity by less than $0.1 \%$. A lattice spacing of $\Delta x=0.125 / \eta$ and a time step of $\Delta t=0.04 \Delta x / u$ were found to supply similar accuracy. To ensure that $\partial c / \partial x=0$ at $x= \pm 1$, the value of $c( \pm 1, z, t)$ is chosen so that a parabolic fit to $c( \pm 1 \mp \Delta x, z, t)$ and $c( \pm 1 \mp 2 \Delta x, z, t)$ reaches its minimum at $x= \pm 1$, for each value of $z$.

Our finite-difference form of Eq. (8) employs a forward difference for the time derivative and a central difference for the first spatial derivative, thereby allowing the lattice values of the concentration at each time step to be obtained explicitly from the previous values. As the concentration profile relaxes to its asymptotic form starting from the initial condition (23), successive estimates of the front velocity are found by computing the average velocity of the front as it propagates vertically a distance equal to the gap width (a vertical distance of 2 in dimensionless units). The front is considered to have relaxed to its asymptotic form when the absolute value of the difference between successive velocity estimates is less than 0.001 . This process is found to converge especially rapidly for large $\eta$, although larger $\eta$ demands larger lattice sizes to adequately resolve the front. The practical limits on the range of our numerical calculations, $1 \leq \eta$ $\leq 16$, are set by slow convergence for $\eta \rightarrow 0$ and by large lattices for $\eta \rightarrow \infty$.

\section{RESULTS AND CONCLUSIONS}

Figure 1 shows constant-concentration front profiles $z$ $=h_{c}(x)$ obtained numerically for $\eta=2$ (a) and $\eta=8$ (b) for

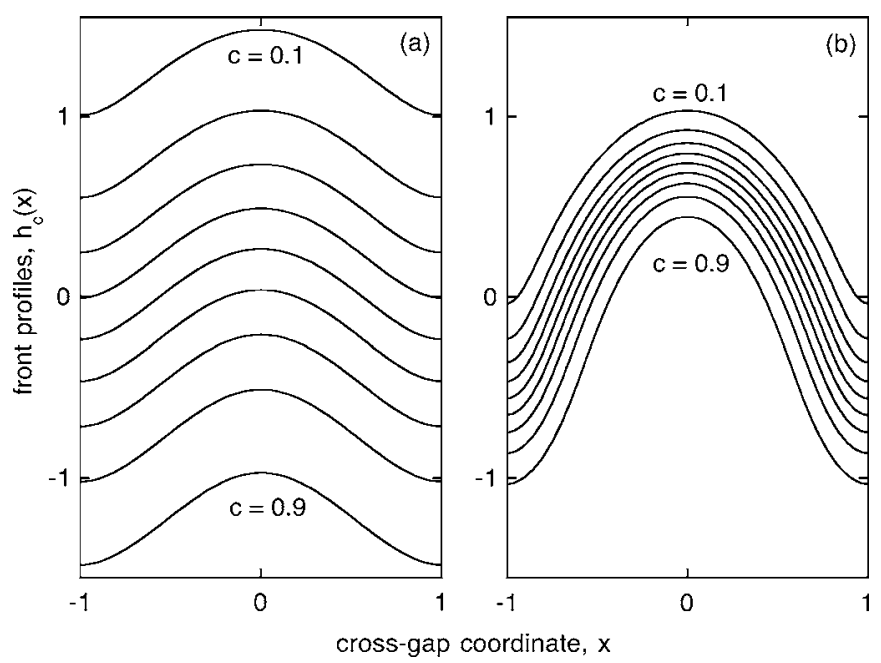

FIG. 1. Dimensionless constant-concentration front profiles $z=h_{c}(x)$ for an autocatalytic reaction front confined between parallel plates vs the dimensionless cross-gap coordinate $x$, for concentrations $c=0.1,0.2,0.3,0.4,0.5$, $0.6,0.7,0.8$, and 0.9 . The concentrations $c=0$ and $c=1$ are respectively achieved far ahead of the front, in the unreacted fluid at $z \rightarrow \infty$, and far behind the front, in the fully reacted fluid at $z \rightarrow-\infty$. The front is advected and distorted by supportive Poiseuille flow with dimensionless gap-averaged velocity $\epsilon=2$ in the same direction as the chemical reaction $(+z)$. Shown are profiles for gap half width to front thickness ratios $\eta=2$ (a) and $\eta=8$ (b), with front velocities $u=3.14$ and 3.70 , respectively.

supportive Poiseuille flow with $\epsilon=2$. For this figure, the fluid flow is in the $+z$ direction, the direction that the chemical reaction front would propagate through a static fluid. Larger $\eta$ implies a thinner front (more closely spaced contours) relative to the gap width, a diminished role of lateral molecular diffusion, and greater front distortion (difference between the vertical and horizontal extents of a single contour). The associated increased front surface area allows the reaction to consume more fluid, and leads to a higher front propagation velocity. The high curvatures and front thickening near the $x= \pm 1$ boundaries for $\eta=8$ anticipate the singular behavior at these boundaries for $\eta \rightarrow \infty$. Compared with Fig. 3 of Ref. 8, Fig. 1 shows enough detail in the concentration profiles to reveal this front thickening, which results from the high positive curvature and correspondingly high catalyst concentrations near $x= \pm 1$, advancing the $c=0.1$ profile relative to the other profiles and thereby thickening the front.

Figure 2 shows constant-concentration front profiles $z$ $=h_{c}(x)$ obtained numerically for $\eta=2$ (a) and $\eta=8$ (b) for adverse Poiseuille flow with $\epsilon=-2$. For this figure, the flow is in the $-z$ direction, the direction opposite the direction that the chemical reaction front would propagate through a static fluid. Again, larger $\eta$ implies a thinner front and greater front distortion. In contrast with Fig. 1, however, the high curvatures and front thickening occur near $x=0$ for $\eta=8$, anticipating the midgap singularity for $\eta \rightarrow \infty$. Midgap front thickening occurs because high curvatures in the leading $c=0.1$ and $c=0.2$ profiles imply increased midgap catalyst concentrations, which advance these profiles in the $+z$ direction and reduce their curvatures. Adverse Poiseuille flow dominates over the chemical reaction for $\eta=2$, driving the front in the direction of the flow. The chemical reaction dominates for $\eta=8$, overwhelming the flow and allowing the front to 


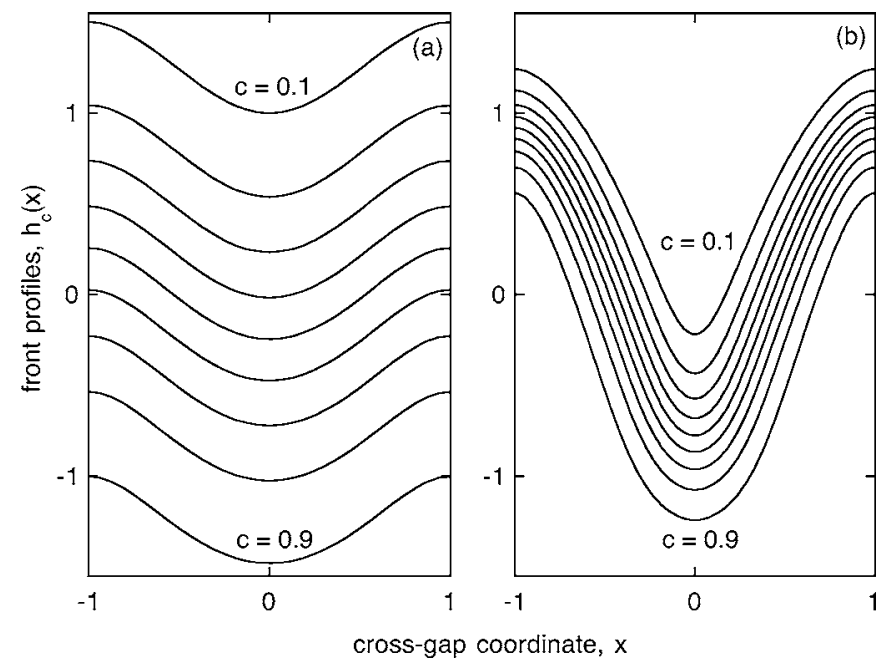

FIG. 2. Front profiles $z=h_{c}(x)$ similar to Fig. 1, except that the Poiseuille flow is in the adverse $-z$ direction, with dimensionless gap-averaged velocity $\epsilon=-2$. Again shown are profiles for $\eta=2$ (a) and $\eta=8$ (b), but now with front velocities $u=-0.869$ and 0.020 , respectively. For $\eta=2$, the flow overwhelms the front, and carries it in the direction opposite its natural direction of propagation. For $\eta=8$, significant front distortion increases the overall fluid consumption rate, and thereby allows the front to propagate in its natural direction of propagation, which is opposite to the flow.

propagate in the direction that it would have propagated through a static fluid, albeit at a much reduced velocity. See Fig. 4 of Ref. 8 for traces for other values of $\eta$, including $\eta \rightarrow \infty$.

The dimensionless front velocity reduces to $u=1+\epsilon$ in both the $\epsilon \rightarrow 0$ and $\eta \rightarrow 0$ limits. Accordingly, the same value of the front velocity applies for both small gaps and smallamplitude flows. In the singular $\eta \rightarrow \infty$ limit, the slope of $u$ vs $\epsilon$ is discontinuous at $\epsilon=0 ; u=1$ for $\epsilon<0$ and $u=1$ $+3 \epsilon / 2$ for $\epsilon>0$. This slope is continuous for all finite $\eta$. To examine the detailed $\eta$ dependence of our general numerical results for $u$, we define velocity corrections $f(\eta, \epsilon)$ and $g(\eta, \epsilon)$ by

$$
u=1+\epsilon+\frac{\epsilon}{2} f(\eta, \epsilon)
$$

for $\epsilon>0$, and

$$
u=1+\epsilon-\epsilon g(\eta, \epsilon)
$$

for $\epsilon<0$. To reflect the correct asymptotic behaviors of $u$ for $\epsilon \rightarrow 0, \eta \rightarrow 0$, and $\eta \rightarrow \infty$, these corrections must satisfy

$$
f(0, \epsilon)=f(\eta, 0)=g(0, \epsilon)=g(\eta, 0)=0
$$

and

$$
f(\infty, \epsilon)=g(\infty, \epsilon)=1 .
$$

The data points (circles, squares, diamonds, crosses, and pluses) in Figs. 3 and 4 give our numerical results for these corrections, for $|\epsilon|=0.5,1,2,4$, and 8 , respectively. The convergence of these results to the asymptotic results given by Eqs. (55) and (56) numerically verifies the analytical limits on $u$, which are summarized at the beginning of this paragraph. Evidently, $u$ increases monotonically with increasing $\eta$ at fixed $\epsilon$, and larger values of $|\epsilon|$ have faster approaches to

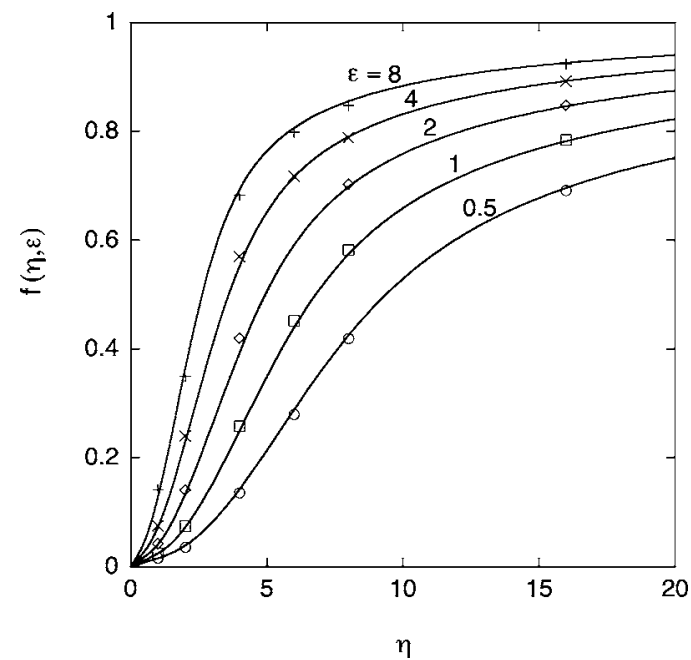

FIG. 3. Front velocity correction $f(\eta, \epsilon)=2(u-1-\epsilon) / \epsilon$ vs $\eta$ for $\epsilon=0.5,1,2$, 4 , and 8. Data points are from the numerical simulations, and solid traces are given by Eq. (57), an empirical fit to the data.

the $\eta \rightarrow \infty$ results. Thus, for increasing gap width, maximum front velocities are achieved more quickly for largeamplitude Poiseuille flows than for small-amplitude flows.

For the purpose of supporting future investigations of nonlinear fronts, we have constructed the empirical functions

$$
f(\eta, \epsilon)=\frac{1+f_{1} e^{-f_{2} / \eta}}{\left[\left(1+f_{1}\right)^{4}+\left(f_{3} \eta\right)^{-4}\right]^{1 / 4}},
$$

with $f_{1}=5.7 \epsilon^{-0.28}, f_{2}=4.6 \epsilon^{-0.50}$, and $f_{3}=0.023 \epsilon^{0.59}$, and

$$
g(\eta, \epsilon)=\frac{2}{1+e^{g_{1} \eta^{-0.8}}},
$$

with $g_{1}=7.5|\epsilon|^{-0.34}$. These functions (solid traces in Figs. 3 and 4) agree with our numerical data to within a few percent over the entire computed range.

Our results are applicable to investigations of nonlinear fronts propagating upward through gaps exceeding the gap

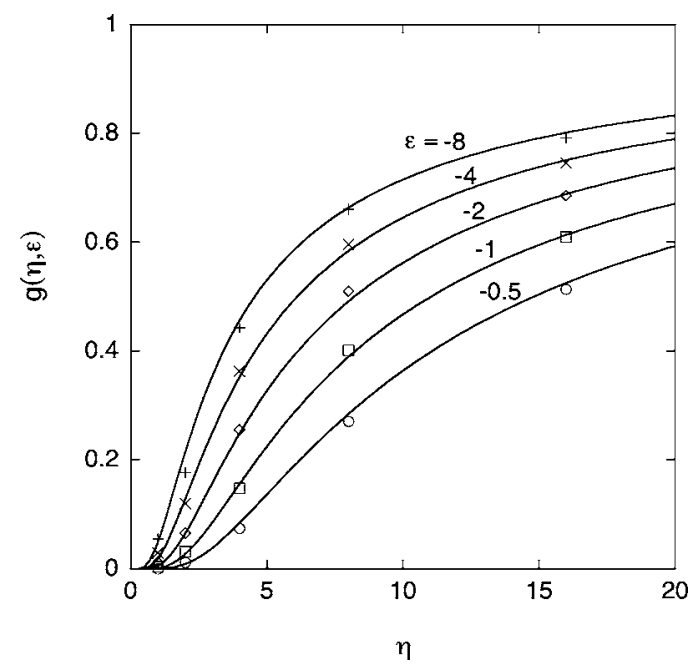

FIG. 4. Front velocity correction $g(\eta, \epsilon)=1+(1-u) / \epsilon$ vs $\eta$ for $\epsilon=-0.5,-1$, $-2,-4$, and -8 . Data points are from the numerical simulations, and solid traces are given by Eq. (58), an empirical fit to the data. 
threshold for the onset of convection., ${ }^{2,3,9-12}$ These fronts are unstable, and quickly assume complicated fingered shapes in the $y-z$ plane, for which the direction $\hat{p}$ of propagation and the component $W=\hat{p} \cdot \overline{\mathbf{V}}$ of the average fluid velocity in this direction vary from point to point along the front. For the purpose of investigating such fronts, the effects of front thickness and distortion in the $x-z$ plane might best be ignored in favor of the larger scale motion in the $y-z$ plane. Such neglect is justified as long as the length scale $L$ of the motion in the $y-z$ plane is large compared with the gap width $a$ and with the front thickness $L_{0}$. Under these conditions, the front, though evolving with time in the $y-z$ plane, can be considered to be quasisteady in the sense that the relationship between the local front velocity and the local flow velocity obeys the steady-state relations given by Eqs. (53)-(58) at all times. Although a time $a / U_{0}$ is actually required for the local front velocity to respond to changes in the local flow velocity, this time is small compared with the time $L / U_{0}$ required for changes in the large-scale structure of the $y-z$ profile of the front. Under these quasisteady conditions, the unit vector $\hat{n}$ normal to the front at midgap $(x=0)$ coincides with the direction $\hat{p}$ of propagation of the front because the front is even about $x=0$.

The fluid velocity $\mathbf{V}$ may be replaced by its gap average $\overline{\mathbf{V}}$ and the $x$ dependence of $C$ may be ignored in Eq. $(1)^{2,3}$ when $f(\eta, \epsilon)$ and $g(\eta, \epsilon)$ are small. Under these conditions, inserting Eqs. (53) and (54) into the local front velocity $U$ $=U_{0} u$ gives $U=U_{0}+W$. For problems involving both supportive and adverse Poiseuille flows of comparable amplitudes, the supportive flows will lead to corrections to $U$ $=U_{0}+W$ for any finite $\epsilon$ and $\eta$, since $\partial f(\eta, \epsilon) / \partial \eta>0$ as $\eta$ $\rightarrow 0$. According to Eqs. (53) and (57), such corrections are of approximate relative order $\epsilon f / 2 \approx 0.01 \epsilon^{1.6} \eta$ for small $\eta$ $=a / 2 L_{0}$ and $\epsilon=W / U_{0}$. For the typical values $a=0.5 \mathrm{~mm}$, $L_{0}=0.07 \mathrm{~mm}$, and $U_{0}=0.03 \mathrm{~mm} / \mathrm{s},{ }^{9}$ gap-averaged flow velocities of at most $|W|=0.02 \mathrm{~mm} / \mathrm{s}$ result in corrections of at most $2 \%$.

When the departures from $U=U_{0}+W$ are not negligible, calculations may simply employ Eqs. (53)-(58) to compute the local front velocity $U=u U_{0}$ based on the local dimensionless flow velocity $\epsilon=W / U_{0}$ at each point along the front. This procedure avoids the need to resolve the reaction front across the gap during complicated computations.

There is a need to evaluate the role of departures from Poiseuille flow, which may become important for wide gaps and ascending fronts, for which fluid buoyancy drives the convective instability. There may also be small departures from Poiseuille flow for descending fronts, since distortion of the front raises the overall gravitational potential energy. Evaluations of such departures might be initiated either theoretically or experimentally.

Experiments for gravitationally stable fronts (descending iodate-arsenous acid fronts, or ascending chloritetetrathionate fronts) might be valuable, since departures from
Poiseuille flow are expected to be small. Such experiments would help to evaluate the theory presented here, and might be particularly simple to carry out, especially when the narrow slab geometry is replaced by a cylindrical geometry.

\section{ACKNOWLEDGMENTS}

Helpful discussions and correspondence with M. Boeckmann, A. De Wit, W. F. Edwards, E. Reynolds, K. Showalter, R. Spangler, D. Vasquez, and J. Wilder are gratefully acknowledged.

${ }^{1}$ A. Saul and K. Showalter, in Oscillations and Traveling Waves in Chemical Systems, edited by R. J. Field and M. Burger (Wiley, New York, 1985), p. 419; A. Hanna, A. Saul, and K. Showalter, J. Am. Chem. Soc. 104, 3838 (1982).

${ }^{2}$ J. Huang and B. F. Edwards, Phys. Rev. E 54, 2620 (1996)

${ }^{3}$ A. De Wit, Phys. Rev. Lett. 87, 054502 (2001).

${ }^{4}$ D. Horváth, T. Bánsági, and Á. Tóth, J. Chem. Phys. 117, 4399 (2002); T. Bánsági, D. Horváth, and Á. Tóth, Phys. Rev. E 68, 026303 (2003); T. Bánsági, D. Horváth, Á. Tóth, J. Yang, S. Kalliadasis, and A. De Wit, Phys. Rev. E 68, 055301(R) (2003); T. Bánsági, D. Horváth, and Á. Tóth, J. Chem. Phys. 121, 11912 (2004); T. Rica, D. Horváth, and Á. Tóth, Chem. Phys. Lett. 408, 422 (2005).

${ }^{5}$ J. Yang, A. D'Onofrio, S. Kalliadasis, and A. De Wit, J. Chem. Phys. 117, 9395 (2002); S. Kalliadasis, J. Yang, and A. De Wit, Phys. Fluids 16, 1395 (2004)

${ }^{6}$ M. Leconte, J. Martin, N. Rakotomalala, D. Salin, and Y. C. Yortsos, J. Chem. Phys. 120, 7314 (2004).

${ }^{7}$ L. D. Landau and E. M. Lifshitz, Course of Theoretical Physics: Fluid Mechanics (Pergamon, New York, 1987), Chap. II.

${ }^{8}$ B. F. Edwards, Phys. Rev. Lett. 89, 104501 (2002).

${ }^{9}$ M. Böckmann and S. C. Müller, Phys. Rev. Lett. 85, 2506 (2000).

${ }^{10}$ D. A. Vasquez, J. W. Wilder, and B. F. Edwards, J. Chem. Phys. 104, 9926 (1996).

${ }^{11}$ M. R. Carey, S. W. Morris, and P. Kolodner, Phys. Rev. E 53, 6012 (1996).

${ }^{12}$ J. Huang, D. A. Vasquez, B. F. Edwards, and P. Kolodner, Phys. Rev. E 48, 4378 (1993).

${ }^{13}$ D. A. Vasquez, Phys. Rev. E 56, 6767 (1997).

${ }^{14}$ D. A. Vasquez and C. Lengacher, Phys. Rev. E 58, 6865 (1998).

${ }^{15}$ J. Martin, N. Rakotomalala, D. Salin, and M. Böckmann, Phys. Rev. E 65, 051605 (2002).

${ }^{16}$ J. J. Tyson and J. P. Keener, Physica D 32, 327 (1988).

${ }^{17}$ B. F. Edwards, J. W. Wilder, and K. Showalter, Phys. Rev. A 43, 749 (1991).

${ }^{18}$ R. S. Spangler and B. F. Edwards, J. Chem. Phys. 118, 5911 (2003).

${ }^{19}$ M. Leconte, J. Martin, N. Rakotomalala, and D. Salin, Phys. Rev. Lett. 90, 128302 (2003).

${ }^{20}$ A. De Wit and G. M. Homsy, Phys. Fluids 11, 949 (1999); A. De Wit and G. M. Homsy, J. Chem. Phys. 110, 8663 (1999).

${ }^{21}$ R. Aris, Proc. R. Soc. London, Ser. A 235, 67 (1956).

${ }^{22}$ M. Diewald, K. Matthiessen, S. C. Müller, and H. R. Brand, Phys. Rev. Lett. 77, 4466 (1996).

${ }^{23}$ K. Matthiessen, H. Wilke, and S. C. Müller, Phys. Rev. E 53, 6056 (1996).

${ }^{24}$ H. Wilke, Physica D 86, 508 (1995)

${ }^{25}$ J. Martin, N. Rakotomalala, and D. Salin, Phys. Fluids 14, 902 (2002).

${ }^{26}$ M. C. Rogers and S. W. Morris, Phys. Rev. Lett. 95, 024505 (2005).

${ }^{27}$ D. A. Vasquez, J. W. Wilder, and B. F. Edwards, J. Chem. Phys. 98, 2138 (1993).

${ }^{28} \mathrm{~J}$. Kevorkian and J. D. Cole, Perturbation Methods in Applied Mathematics (Springer, New York, 1981), p. 28; see also Philipp Kuegler, http:// www.tbi.univie.ac.at/ svrci/Leere/PDF/singpert-pp4.pdf, 1-11 (2005, accessed May 30, 2006). 